\title{
HIGHER FANO MANIFOLDS AND RATIONAL SURFACES
}

\author{
A. J. DE JONG AND JASON STARR
}

\begin{abstract}
Let $X$ be a Fano manifold of pseudo-index $\geq 3$ such that $c_{1}(X)^{2}-$ $2 c_{2}(X)$ is nef. Irreducibility of some spaces of rational curves on $X$ (in fact, a weaker hypothesis) implies a general point of $X$ is contained in a rational surface.
\end{abstract}

\section{INTRODUCTION}

One consequence of the bend-and-break lemma is uniruledness of Fano manifolds, MM86. In fact, in characteristic 0, Fano manifolds are rationally connected, KMM92, Cam92. We prove an analogous theorem with rational curves replaced by rational surfaces for Fano manifolds satisfying positivity of the second graded piece of the Chern character.

Definition 1.1. A Fano manifold is 2-Fano if $\operatorname{ch}_{2}\left(T_{X}\right)$ is nef, where $\operatorname{ch}_{2}\left(T_{X}\right)$ is the second graded piece of the Chern character, $\frac{1}{2}\left(c_{1}\left(T_{X}\right)^{2}-2 c_{2}\left(T_{X}\right)\right)$. In other words, $\operatorname{deg}\left(\left.\operatorname{ch}_{2}\left(T_{X}\right)\right|_{S}\right)$ is nonnegative for every surface $S$ in $X$.

Let $\mathcal{M}$ be a positive-dimensional, irreducible component of the Artin stack $\overline{\mathcal{M}}_{0,0}(X)$ of genus 0 stable maps to $X$ whose general point of $\mathcal{M}$ parametrizes a stable map with irreducible domain. Denote by $M$ the coarse moduli space of $M$. Denote by $\Delta$ the locally principal closed substack of $\overline{\mathcal{M}}_{0,0}(X)$ parametrizing stable maps with reducible domain. The closed substack $\mathcal{M} \cap \Delta$ is a Cartier divisor. The question we consider is uniruledness of $M$.

Theorem 1.2. If $X$ is 2-Fano, every point of $M$ parametrizing a free curve and contained in a proper curve in $M-M \cap \Delta$ is contained in a rational curve in $M$.

If a general point of $M$ parametrizes a birational, free curve and is contained in a proper curve in $M-M \cap \Delta$, then a general point of $X$ is contained in a rational surface.

The proof uses the bend-and-break approach of [MM86]. Given a general curve $C$ in $M$, we need to bound the dimension of $\operatorname{Hom}(C, M)$ from below. Although $M$ and $\mathcal{M}$ may be very singular, the deformation theory of stable maps nonetheless gives a useful lower bound. This uses Grothendieck-Riemann-Roch computations from dJS05a. Unfortunately, the formula has a negative term coming from intersection points of $C$ and $\Delta$. This is the reason for the hypothesis that $M-M \cap \Delta$ contains a proper curve. Luckily, there are nice sufficient conditions for $M-M \cap \Delta$ to contain many proper curves.

Proposition 1.3. If the pseudo-index of $X$ is $\geq 3$ and every irreducible component of $\mathcal{M} \cap \Delta$ is an irreducible component of $\Delta$, then $M-M \cap \Delta$ is a union of proper curves. 
This uses a contraction of the locally principal closed subspace $\Delta$ in $\overline{\mathcal{M}}_{0,0}(X)$ discovered in [CHS05] and independently by Adam Parker Par05.

Section 3 gives some examples of 2-Fano manifolds and makes some observations about classification. Section 4 shows Theorem 1.2 is sharp in 2 ways. First, there are Fano manifolds that are not 2-Fano where the components $\mathcal{M}$ are not uniruled. Second, there are 2-Fano manifolds where the components $\mathcal{M}$ are uniruled but not rationally connected. Finally Section 5 speculates on sufficient conditions for the components $\mathcal{M}$ to be rationally connected.

\section{Proof of the theorem}

For every point $x$, denote by $\operatorname{Hom}\left(\mathbb{P}^{1}, X, 0 \mapsto x\right)_{\text {nc }}$ the open subscheme of $\operatorname{Hom}\left(\mathbb{P}^{1}, X, 0 \mapsto x\right)$ parametrizing nonconstant morphisms.

Lemma 2.1. The dimension of every irreducible component of $\operatorname{Hom}\left(\mathbb{P}^{1}, X, 0 \mapsto\right.$ $x)_{n c}$ is at least as large as the pseudo-index of $X$.

Proof. This follows from Kol96, Theorem II.1.2, Corollary II.1.6].

Proof of Proposition 1.3, Let $f: X \hookrightarrow \mathbb{P}^{N}$ be a plurianticanonical embedding. Denote by $\overline{\mathcal{M}}_{0,0}(f): \overline{\mathcal{M}}_{0,0}(X) \rightarrow \overline{\mathcal{M}}_{0,0}\left(\mathbb{P}^{N}\right)$ the associated embedding. Denote by $\phi: \overline{\mathcal{M}}_{0,0}\left(\mathbb{P}^{N}\right) \rightarrow Y$ the contraction of the boundary constructed in CHS05]. Denote by $N$ the image of $\mathcal{M}$ in $Y$.

Since the restriction of $\phi$ to $\overline{\mathcal{M}}_{0,0}\left(\mathbb{P}^{N}\right)-\Delta$ is an open immersion, the restriction of $\phi \circ \overline{\mathcal{M}}_{0,0}(f)$ to $\mathcal{M}-\Delta$ is and immersion. Since $\mathcal{M}_{\text {free }}$ is dense in $\mathcal{M}, \mathcal{M}$ has pure dimension equal to the expected dimension, and $\mathcal{M} \cap \Delta$ is a Cartier divisor. Therefore $\operatorname{dim}(N)$ equals $\operatorname{dim}(M)$ and $\operatorname{dim}(\mathcal{M} \cap \Delta)$ equals $\operatorname{dim}(M)-1$.

If $i \leq j$, the restriction of $\phi$ to the boundary divisor $\Delta_{i, j}$ factors through the projection $\pi_{j}: \Delta_{i, j} \rightarrow \overline{\mathcal{M}}_{0,1}\left(\mathbb{P}^{N}, j\right)$. Denote $\Delta_{i, j} \cap \overline{\mathcal{M}}_{0,0}(X)$ by $\Delta_{X, i, j}$. Denote the restriction of $\pi_{j}$ by $\pi_{X, j}: \Delta_{X, i, j} \rightarrow \overline{\mathcal{M}}_{0,1}\left(\mathbb{P}^{N}, j\right)$. By Lemma 2.1$]$ every irreducible component of every fiber of $\pi_{X, j}$ has dimension $\geq 1$, i.e., the difference of the pseudo-index and $\operatorname{dim}\left(\operatorname{Aut}\left(\mathbb{P}^{1}, 0\right)\right)$. Therefore, for every irreducible component $\Delta^{\prime}$ of $\Delta$, the dimension of $\phi\left(\overline{\mathcal{M}}_{0,0}(f)\left(\Delta^{\prime}\right)\right)$ is strictly less than the dimension of $\Delta^{\prime}$. By hypothesis, every irreducible component $\Delta^{\prime}$ of $\mathcal{M} \cap \Delta$ is an irreducible component of $\Delta$. Since $\operatorname{dim}\left(\Delta^{\prime}\right)$ equals $\operatorname{dim}(\mathcal{M})-1$, the image of $\Delta^{\prime}$ in $N$ has dimension $\leq \operatorname{dim}(N)-2$.

Since every connected component of $Y$ is projective, also $N$ is projective. Because $\operatorname{dim}\left(\operatorname{Image}\left(\Delta^{\prime}\right)\right) \leq \operatorname{dim}(N)-2$, a general intersection of $N$ with $\operatorname{dim}(N)-1$ hyperplanes containing a point of $N-\operatorname{Image}\left(\Delta^{\prime}\right)$ is a complete curve that does not intersect Image $\left(\Delta^{\prime}\right)$. Because there are only finitely many irreducible components of $\mathcal{M} \cap \Delta$, a general intersection of $N$ with $\operatorname{dim}(N)-1$ hyperplanes containing a point of $N$ - Image $(\mathcal{M} \cap \Delta)$ is a complete curve that does not intersect Image $(\mathcal{M} \cap \Delta)$. The inverse image of this curve in $\mathcal{M}-\mathcal{M} \cap \Delta$ is a complete curve containing a given point of $\mathcal{M}-\mathcal{M} \cap \Delta$.

Let $C$ be a smooth, proper, connected curve and let $\zeta: C \rightarrow \overline{\mathcal{M}}_{0,0}(X)-\Delta$ be a nonconstant 1-morphism whose general point parametrizes a free curve of $\left(-K_{X}\right)$ degree $e$. Let $B$ be a finite set of closed points of $C$. Denote by $(\pi: \Sigma \rightarrow C, F$ : $\Sigma \rightarrow X)$ the associated family of stable maps. 
Lemma 2.2. The dimension at $[\zeta]$ of $\operatorname{Hom}\left(C, \overline{\mathcal{M}}_{0,0}(X),\left.\zeta\right|_{B}\right)$ is at least,

$$
\operatorname{deg}\left(\left.\operatorname{ch}_{2}\left(T_{X}\right)\right|_{F(\Sigma)}\right)+\frac{1}{2 e} \operatorname{deg}\left(\left.c_{1}\left(T_{X}\right)^{2}\right|_{F(\Sigma)}\right)+(e+\operatorname{dim}(X)-3)(1-g(C)-\#(B)) .
$$

Proof. Consider the finite morphism $(\pi, g): \Sigma \rightarrow C \times X$. Denote by $\mathcal{N}$ the cokernel of the map,

$$
d(\pi, g): T_{\Sigma} \rightarrow \pi^{*} T_{C} \oplus g^{*} T_{X} .
$$

By a natural generalization of $\underline{\text { Kol96, }}$, Theorem I.2.16], the dimension of $\operatorname{Hom}\left(C, \overline{\mathcal{M}}_{0,0}(X),\left.\zeta\right|_{B}\right)$ at $\zeta$ is at least,

$$
h^{0}(\Sigma, \mathcal{N})-h^{1}(\Sigma, \mathcal{N}) .
$$

By the Leray spectral sequence, $h^{2}(\Sigma, \mathcal{N})$ equals $h^{1}\left(C, R^{1} \pi_{*} \mathcal{N}\right)$. Because a general point of $C$ parametrizes a free curve, the restriction of $\mathcal{N}$ to a general fiber of $\pi$ is generated by global sections, thus has no higher cohomology. Thus $R^{1} \pi_{*} \mathcal{N}$ is a torsion sheaf so that $h^{1}\left(C, R^{1} \pi_{*} \mathcal{N}\right)$ is 0 . Therefore, the lower bound actually equals $\chi(\Sigma, \mathcal{N})$.

Finally, by the Grothendieck-Riemann-Roch computations from dJS05a, $\chi(\Sigma, \mathcal{N})$ equals,

$$
\operatorname{deg}\left(\left.\operatorname{ch}_{2}\left(T_{X}\right)\right|_{F(\Sigma)}\right)+\frac{1}{2 e} \operatorname{deg}\left(\left.c_{1}\left(T_{X}\right)^{2}\right|_{F(\Sigma)}\right)+(e+\operatorname{dim}(X)-3)(1-g(C)-\#(B)) .
$$

Proof of Theorem 1.2. Every proper curve in $M-M \cap \Delta$ is the image of a nonconstant 1-morphism $\zeta: C \rightarrow \mathcal{M}-\mathcal{M} \cap \Delta$ from a smooth curve $C$. The induced morphism $\operatorname{Hom}(C, \mathcal{M}-\mathcal{M} \cap \Delta) \rightarrow \operatorname{Hom}(C, M)$ is finite. By Lemma 2.2 $\operatorname{dim}\left(\operatorname{Hom}\left(C, M ;\left.\zeta\right|_{B}\right)\right)$ behaves as if $M$ is smooth along the image of $\zeta$ and the anticanonical degree of $\zeta(C)$ equals

$$
\operatorname{deg}\left(\left.\operatorname{ch}_{2}\left(T_{X}\right)\right|_{F(\Sigma)}\right)+\frac{1}{2 e} \operatorname{deg}\left(\left.c_{1}\left(T_{X}\right)^{2}\right|_{F(\Sigma)}\right) .
$$

Because $X$ is 2-Fano, this degree is positive. Therefore the usual bend-and-break argument applies, cf. Kol96, Theorem II.5.8].

\section{EXAMPles Of 2-FANO MANifoldS}

All the results of this section, and more, are discussed and proved in the note dJS05b. There are a two families of 2-Fano manifolds. The first family comes from complete intersections. Let $\mathbb{P}$ be a weighted projective space of dimension $n$. Let $X \subset \mathbb{P}$ be a smooth complete intersection of type $\left(d_{1}, \ldots, d_{r}\right)$. Then $X$ is Fano if and only if $d_{1}+\cdots+d_{r} \leq n$. It is 2-Fano if and only if $d_{1}^{2}+\cdots+d_{r}^{2} \leq n$.

The second family comes from Grassmannians. Let $\mathbb{G}$ be a Grassmannian $\operatorname{Grass}(k, n)$ of $k$-dimensional subspaces of a fixed $n$-dimensional vector space. Without loss of generality, assume $n \geq 2 k$. This is Fano. It is 2-Fano if and only if either $k=1, n=2 k$ or $n=2 k+1$.

There are two operations for producing new 2-Fano manifolds. First, if $X$ and $Y$ are each 2-Fano, then the product $X \times Y$ is 2-Fano. The second operation is more interesting. Let $X$ be a smooth Fano manifold and let $L$ be a nef invertible sheaf. The $\mathbb{P}^{1}$-bundle $\mathbb{P}\left(\mathcal{O}_{X} \oplus L^{\vee}\right)$ is Fano if and only if $c_{1}\left(T_{X}\right)-c_{1}(L)$ is ample. Assuming it is Fano, it is 2-Fano if and only if $\operatorname{ch}_{2}\left(T_{X}\right)+\frac{1}{2} c_{1}(L)^{2}$ is nef. Notice, it is not necessary that $\operatorname{ch}_{2}\left(T_{X}\right)$ is nef, i.e., $X$ need not be 2-Fano. 
There are other operations on Fano manifolds. It is reasonable to ask which of these produce 2-Fano manifolds. For instance, a projective bundle $\mathbb{P}(E)$ of fiber dimension $\geq 2$ over a Fano manifold is also Fano if $E$ satisfies a weak version of stability. However, if $\mathbb{P}(E)$ is 2-Fano then the pullback of $E$ to every curve is a semistable bundle. If $X$ is $\mathbb{P}^{n}$, for instance, this implies $\mathbb{P}(E)$ is simply $\mathbb{P}^{m} \times \mathbb{P}^{n}$. This, and other examples, suggest the following principle: an operation on Fano manifolds produces a 2-Fano manifold only if some vector bundle associated to the operation is semistable.

\section{THE THEOREM IS SHARP}

The theorem is sharp in 2 ways. First, let $X$ be a general cubic hypersurface in $\mathbb{P}^{5}$. This is Fano, but it is not 2-Fano. By the main theorem of dJS04, there are infinitely many non-uniruled irreducible components $\mathcal{M}$ of $\overline{\mathcal{M}}_{0,0}(X)$ satisfying the hypotheses of Theorem 1.2

Second, let $Y$ be the $\mathbb{P}^{1}$-bundle over $X, Y=\mathbb{P}\left(\left.\mathcal{O}_{X} \oplus \mathcal{O}_{\mathbb{P}^{5}}(-2)\right|_{X}\right)$. By the construction in the last section, $Y$ is 2-Fano. Associated to the projection $\pi: Y \rightarrow$ $X$, there is a 1-morphism $\overline{\mathcal{M}}_{0,0}(\pi): \overline{\mathcal{M}}_{0,0}(Y) \rightarrow \overline{\mathcal{M}}_{0,0}(X)$. For an irreducible component $\mathcal{N}$ of $\overline{\mathcal{M}}_{0,0}(Y)$ containing a free curve, it is easy to prove the boundary $\mathcal{N} \cap \Delta$ is contracted. (However it is not true that every component of $\mathcal{N} \cap \Delta$ is a component of $\Delta$.) Thus Theorem 1.2 implies $N$ is uniruled. In fact, the restriction of $\overline{\mathcal{M}}_{0,0}(\pi)$ to $\mathcal{N}$ is birational to a projective bundle over the image component $\mathcal{M}$ of $\overline{\mathcal{M}}_{0,0}(X)$. Choosing $\mathcal{N}$ appropriately, $\mathcal{M}$ is one of the infinitely many non-uniruled irreducible components of $\overline{\mathcal{M}}_{0,0}(X)$. Therefore $N$ is not rationally connected, and the MRC quotient of $N$ is precisely $M$.

\section{Speculation}

For the counterexample $Y$ in the previous section, $\operatorname{ch}_{2}\left(T_{Y}\right)$ is nef. But it is not "positive". It has intersection number 0 with the surface $\pi^{-1}(B)$ for every curve $B$ in $X$. If $X$ is a Fano manifold such that $\operatorname{ch}_{2}\left(T_{X}\right)$ has positive intersection number with every surface, is $\mathcal{M}$ rationally connected? We know no counterexample.

\section{REFERENCES}

[Cam92] F. Campana. Connexité rationnelle des variétés de Fano. Ann. Sci. École Norm. Sup. (4), 25(5):539-545, 1992.

[CHS05] Izzet Coskun, Joe Harris, and Jason Starr. The ample cone of the kontsevich moduli space. preprint submitted Trans. Amer. Math. Soc., 2005.

[dJS04] A. J. de Jong and Jason Starr. Cubic fourfolds and spaces of rational curves. Illinois J. Math., 48(2):415-450, 2004.

[dJS05a] A. J. de Jong and J. Starr. Divisor classes and the virtual canonical bundle. preprint, 2005.

[dJS05b] A. J. de Jong and J. Starr. A note on Fano manifolds whose second Chern character is positive. in preparation, 2005.

[KMM92] János Kollár, Yoichi Miyaoka, and Shigefumi Mori. Rational connectedness and boundedness of Fano manifolds. J. Differential Geom., 36(3):765-779, 1992.

[Kol96] János Kollár. Rational curves on algebraic varieties, volume 32 of Ergebnisse der Mathematik und ihrer Grenzgebiete. 3. Folge. A Series of Modern Surveys in Mathematics [Results in Mathematics and Related Areas. 3rd Series. A Series of Modern Surveys in Mathematics]. Springer-Verlag, Berlin, 1996.

[MM86] Yoichi Miyaoka and Shigefumi Mori. A numerical criterion for uniruledness. Ann. of Math. (2), 124(1):65-69, 1986. 
[Par05] Adam Parker. An elementary GIT construction of the moduli space of stable maps. PhD thesis, University of Texas at Austin, 2005. 